\title{
Systematic review on the rational use of amniotic membrane allografts in diabetic foot ulcer treatment
}

\author{
Kasun Lakmal, Oshan Basnayake and D. Hettiarachchi ${ }^{*}$ (D)
}

\begin{abstract}
Background: Diabetic foot ulcer is a complication with multiple aetiological factors which has a significant impact to patients' lives and costs to the healthcare system. The potential of human amniotic membrane to act as an allograft has been studied in relation to this condition. Aim of this study is to evaluate the current scientific evidence on its effectiveness in healing diabetic foot ulcers.

Methods: Pubmed, Cochrane library, and Google scholar were searched using the search terms, "Amnion" OR "Placenta" AND "Diabetic foot". (MeSH terms) in the title or the abstract field from 1st of January 2000 to 30th March 2020. The quality of published reports was assessed using standard methods. We searched for experimental and observational studies in terms of randomized control trials, prospective cohort, retrospective cohort studies and case series.

Results: When searched with Mesh terms, 12 citations in PubMed, 22 citations in Cochrane library and 30 in other data bases were found. After screening the studies and their reference lists, 12 studies met the inclusion criteria and the others were excluded. There were 8 randomized control trials (RCTs), 2 prospective studies and 2 retrospective studies employing different preparation methods of the amniotic membranes. A wide variation in study end points were noted. Majority of the RCTs $(n=7)$ were concluded with significantly higher wound closure rate compared to the conventional treatment groups. In prospective and retrospective studies, it was shown that large chronic ulcers which were resistant to closure with standard therapy achieved wound closure with amniotic membrane allografts. A meta-analysis could not be performed due to study heterogeneity, and publication bias was not assessed due to the small number of available studies which was not sufficient for accurate comparison.
\end{abstract}

Conclusion: Even though, the studies had some inherent heterogeneity due to different preparation methods, different study end points and outcome measurements. According to our review the current studies using amniotic membrane allografts give reliable evidence of reduction in healing time over conventional methods.

Keywords: Amniotic membrane, Diabetes, Foot ulcers, Allografts

\section{Background}

The human amniotic membrane has shown immense potential as an allograft. Owing to its several unique qualities such as a rich milieu of amino acids, growth

*Correspondence: dineshani@anat.cmb.ac.lk

Department of Anatomy, Faculty of Medicine, University of Colombo, 25,

Kynsey Place 8, Colombo, Sri Lanka factors and other nutrients that facilitates its intrauterine function as it forms the feto-maternal interphase. Human Amniotic Allograft Membrane (HAA) can support wound healing by facilitating cell migration and promoting repair [1]. One such use is in the treatment of chronic wounds, in the early twentieth century this possibility was explored and further expanded to diabetic neurovascular ulcers. The recent development of gamma 
irradiated or dehydrated amniotic membrane grafts has enabled us to bypass some of the drawbacks experienced with traditional graphing method including issues with storage and preparation [2].

Diabetic foot ulcers (DFU) are estimated to affect 15\% of diabetic patients. They experience foot ulcers once in their lifetime with a recurrence rate of $35-50 \%$ over 3 years to around $70 \%$ over 5 years [2-4]. Complications of diabetic foot ulcers maybe related to its chronicity, osteomyelitis, re-ulceration, gangrene and amputation which might be aggravated by concomitant co-morbidities such as peripheral vascular disease, sub-optimal blood glucose control and neuropathy to name a few [5]. The long-drawn healing process in a DFU make them more susceptible for infection and resulting complications leading to healthcare economic burden [6]. The standard care for a DFU includes management of infections, local wound care offloading (especially in DFU complicated with neuropathy) and correcting systemic factors to promote healing. Some clinicians recommend advanced treatment such as biological dressings, collagen, platelet-derived growth factors (PDGF), and platelet-rich plasma (PRP) for non-healing ulcers after alstandard wound care [1]. In this light natural amniotic membrane wound dressings have been used for over a century as it contains a single epithelial cell layer, a thick basement membrane and an avascular stroma making it an ideal biological graft. Human amniotic membrane can assist in wound healing by cell migration into the healing tissue. Acquiring placenta for the harvesting of amniotic membrane is a challenge in terms of ethical aspects and the harvesting, processing, and preservation of the membrane as biological dressing are expensive procedures. Products containing amniotic tissue are increasingly being manufactured either as cryopreserved or dehydrated grafts [7]. We sought to investigate the rational use of amniotic membrane allografts in the management of diabetic foot ulcers by conducting a systemic review through published studies. Objective of the study was to assess the impact on wound closure rates by the use of amniotic membrane in diabetic foot ulcers.

\section{Methods}

PubMed, Cochrane library, CINAHL, Embase, Web of Science, and Clinicaltrials.gov and Google scholar engines were searched for the terms "Amnion" OR "Placenta" AND "Diabetic foot" (MeSH terms) in the title or in the abstract field from 1st of January 2000 to $30^{\text {th }}$ March 2020. A non-English language database known as APAMED central was searched using the same criteria to reduce the publication bias. The reference lists provided in full papers were also used to identify additional papers for review. Quality of published reports was assessed using Downs and Black checklist. Downs and Black score ranges were given corresponding quality levels as previously reported [8]: excellent (26-28); good (20-25); fair $(15-19)$; and poor $(\leq 14)$. Additionally authors attempted to reduce the publication bias and between-study heterogeneity by employing standard methods such as extended funnel plot tests for detecting publication bias, and selection modelling and trim-and-fill methods to adjust for publication bias in the presence of between-study heterogeneity.

We searched for experimental and observational studies in terms of randomized control trials, prospective cohorts, and retrospective cohort studies. Case reports were excluded from this review. Only studies pertaining to human subjects were selected. The primary objective of this systematic review was to identify the outcomes of the use of amniotic membrane in the rate of healing in diabetic foot ulcers (Fig. 1).

Initial eligibility screening was performed based on the titles and abstract from electronic databases. Thereafter, the full text papers of all studies were assessed based on the inclusion and exclusion criteria. In doubtful situations the opinion of the senior investigator was sought. The studies done with both type 1 and type 2 diabetes patients were included. The studies which have used different preparation of amniotic allografts (dehydrated, cryopreserved and stem cell extractions) were included. When including the RCTs, studies which compared the amniotic membrane treatment with standard or conventional care were selected. Studies that are designed with the aim of analyzing the molecular basis without measuring clinical improvement of the ulcers were also excluded from our study. From each study data were extracted on trial design, study setting, amniotic membrane preparation methods used, control interventions, outcome measures and statistical analysis. Outcome measures were extracted in terms of the healing time, healed percentage, recurrences and adverse outcomes.

\section{Results}

When searched with Mesh terms 12 citations in Pubmed, 22 citations in Cochrane library and 30 in other data bases were found. We couldn't find new studies by going through reference lists. By screening the studies total of 12 non-duplicated studies met the inclusion and exclusion criteria. There were 8 randomized control trials, 2 prospective studies and 2 retrospective studies (Fig. 1). Even though the search was done from the studies conducted since 2000, all the studies that met the criteria and included in the review were done in the last decade i.e. after 2010. We found 8 randomized control trials [1, 2, 5, 9-13] and all were performed in the United States and five of those were multicenter trials. Out of the 2 


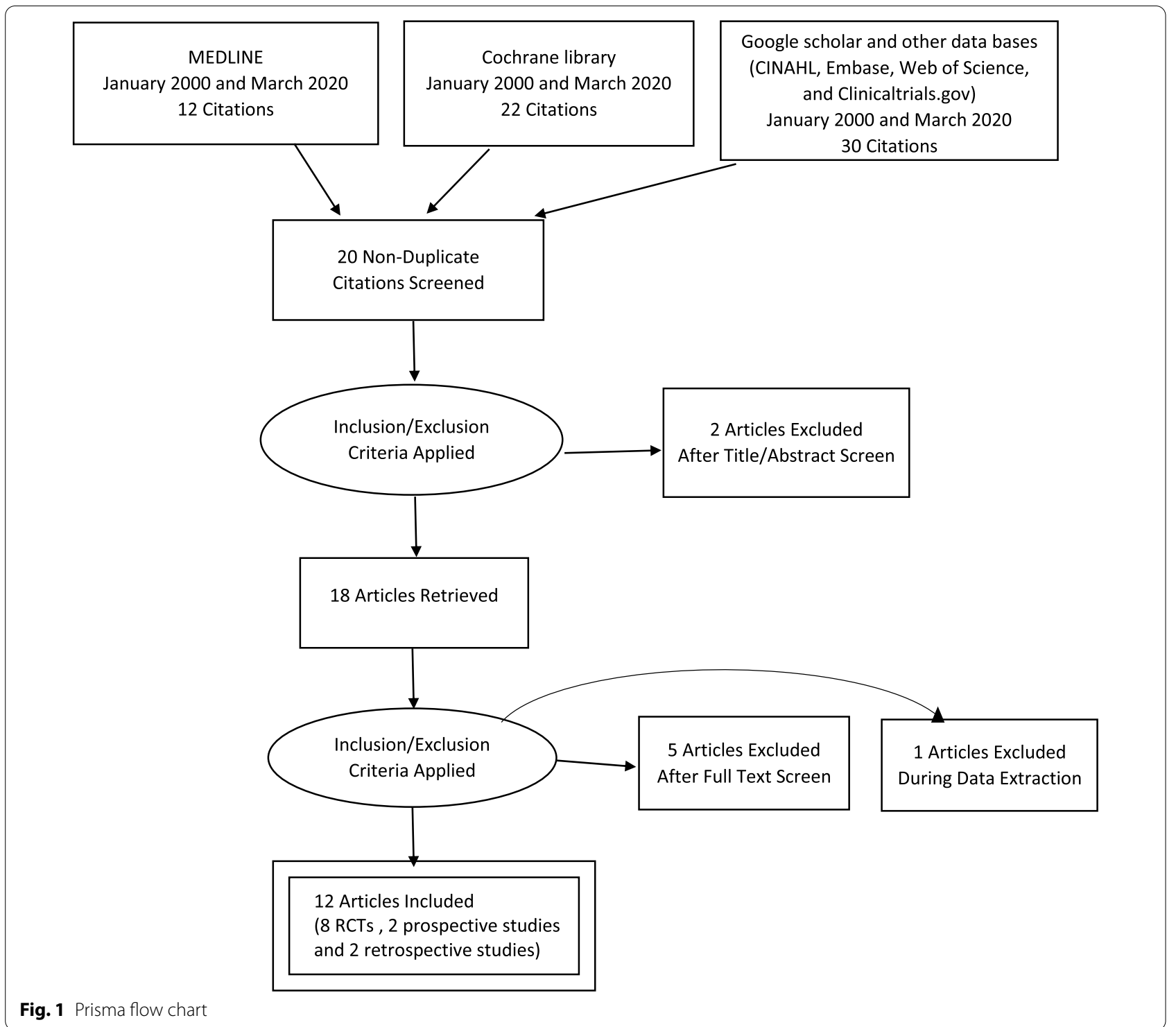

prospective studies one was done in Spain [14] and the other in the United States [15]. Both retrospective studies $[16,17]$ were also performed in the United States. According to the Downs and Black scoring system, 4 studies $[5,9,11,13]$ were graded as "Good" (score ranging from 20 to 25 ) and rest of the 8 studies were graded as "Fair" (15-19).

There were total 244 participants in intervention groups and 210 in the control groups of 8 randomized control trials, except in in one study. Total of 28 in prospective and total of 92 in retrospective studies were treated with amniotic membrane preparations. The mean duration of the diabetes mellitus in the participants was reported only in one study [13]. There were patients with different ulcer locations in their feet and in all the above studies ulcer duration was more than 28 days. Mean size of the ulcers in prospective and retrospective studies were more than $5 \mathrm{~cm}^{2}$ and it was less than $5 \mathrm{~cm}^{2}$ in majority of participants in randomized control trials (Table 1). Different amniotic membrane preparations have been used (Amnioband [9], AMNIOEXCEL [10, 15], Epifix [2,11,12,], Apligraf [11], Grafix [13], NEOX CORD [16], (dHACM) [5, 17]).

In 6 randomized control trials the follow up duration was 12 weeks and in the rest, it was 6 weeks. Both prospective and one retrospective study [16] included data until complete wound closure was achieved. Majority of randomized control trials $(n=7)$ have demonstrated statistically significant closure rates at the study endpoint compared to conventional or standard wound care 


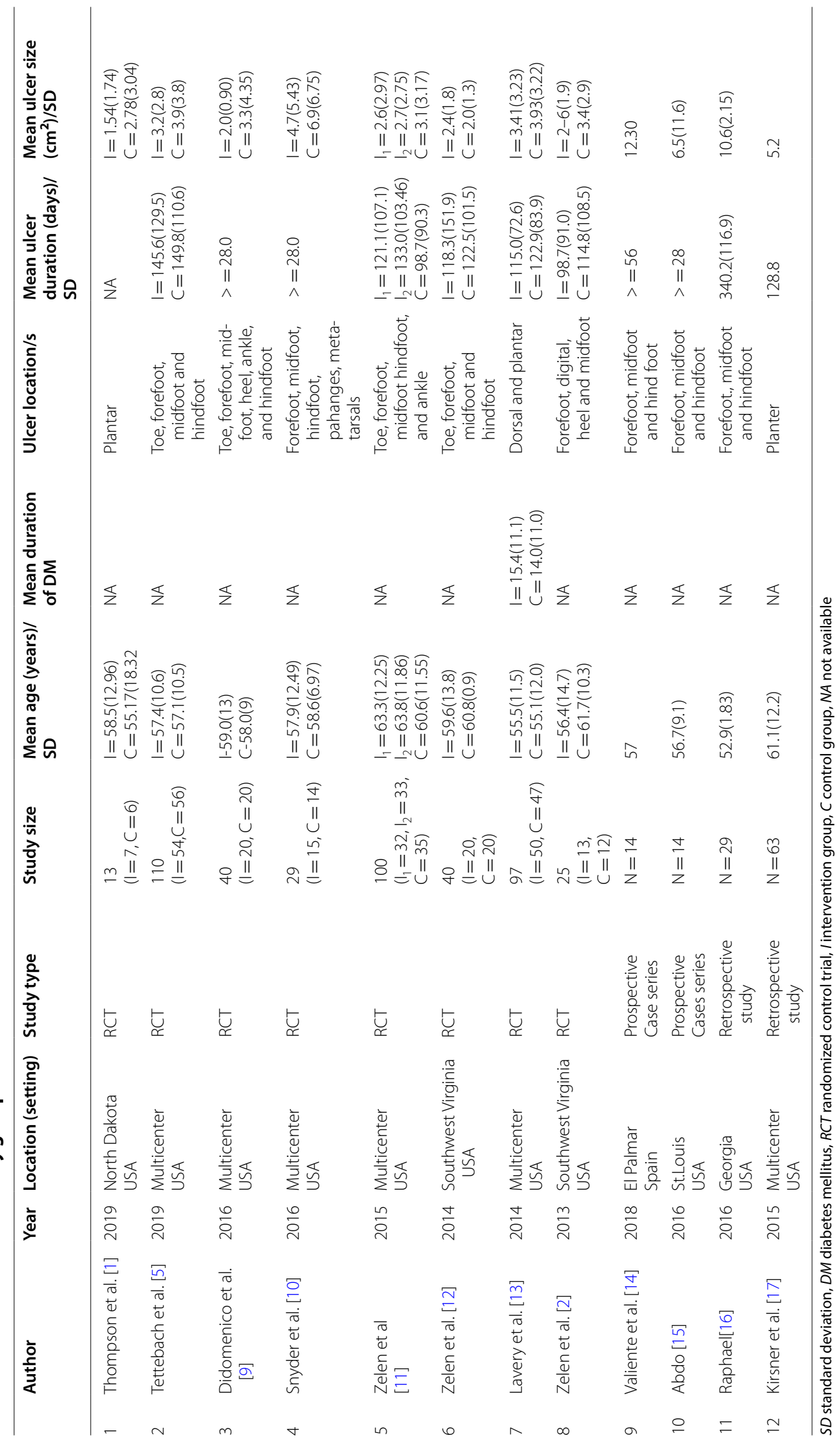




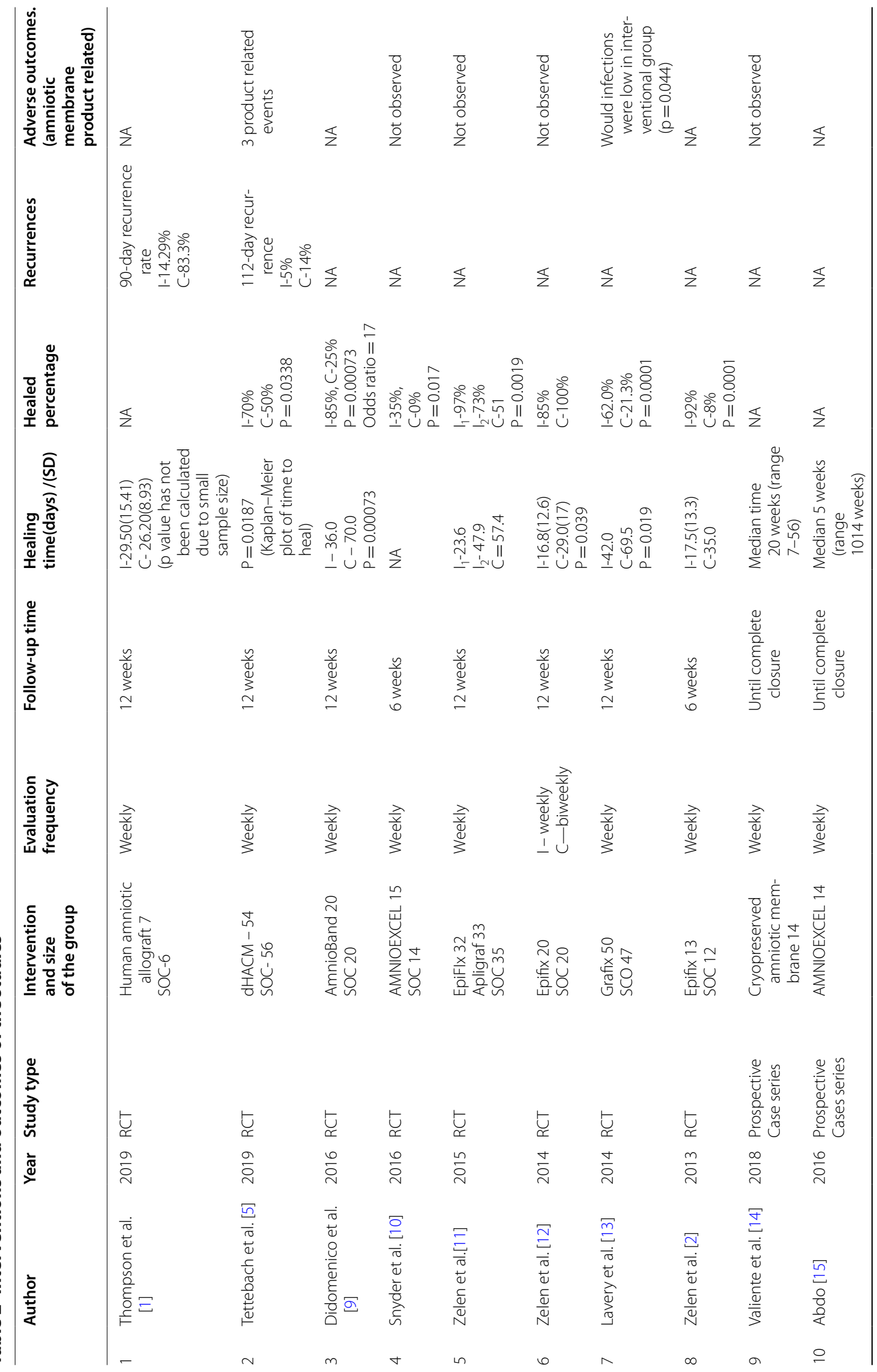




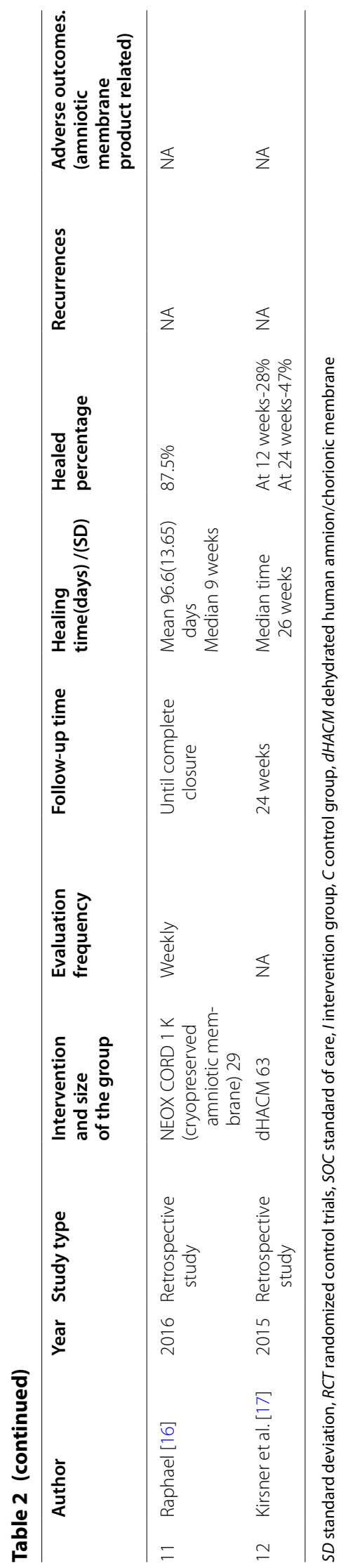


procedures $(\mathrm{p}<0.05)$. Adverse graft outcomes were low in studies where safety evaluation data was available $(n=6)$. One study [13] showed statistically significant low infection rate in the intervention group $(\mathrm{p}<0.044)$. Thompson et al. evaluated 90-day recurrence rates in both intervention and control group and a lower recurrence rate was observed in the intervention group (14.29\% versus $83.3 \%$ ) similarly Tettlebatch et al. also showed a lower recurrence rate at 112 days (5\% versus 14\%).

In one prospective study [14], the mean duration of ulcer was more than 56 days and the mean ulcer size was $12.30 \mathrm{~cm}^{2}$ in comparison the other prospective study [15] these two parameters were more than 28 days and $6.5 \mathrm{~cm}^{2}$. Median ulcer closure times were 20 weeks and 5 weeks, respectively. The mean ulcer duration was longest in one retrospective study [16], which was 340 days with mean ulcer size of $10.6 \mathrm{~cm}^{2}$. This study concluded that the median ulcer closure time was 9 weeks. In the other retrospective study [17], mean ulcer duration was 128.8 days and mean ulcer size was $5.2 \mathrm{~cm}^{2}$ and this study demonstrated that the median time of healing was 26 weeks (Table 2 ).

\section{Discussion}

This study aimed to evaluate the current scientific evidence on effectiveness of use of amniotic membrane in healing the diabetic foot ulcers. In the analysis of retrospective studies, majority of the RCTs $(n=7)$ were concluded with significantly higher wound closure rates compared to the conventional treatment group. One randomized control trial showed less recurrent rate of the healed ulcers after treatment. In prospective and retrospective studies showed that larger and more chronic ulcers which are resistant to close with the standard therapy achieve wound closure with amniotic membrane allografts. Minimal numbers of adverse effects attributable to amniotic membrane product were observed in the included studies.

Only two RCTs aimed at assessing the recurrence rate following total closure of the ulcers [1, 5]. Follow up details were not included in the other studies in terms of recurrent rates and further complications. Amniotic membrane preparations used in different studies were different to each other. Currently commercially available amniotic membranes are expensive and median graft costs in some studies were between 2000 and 10,000 of dollars $[11,12]$. The main limitations of these studies were the heterogeneity study methods and outcomes, limited number of RCTs and small number of the of study participants. Except one study [14] other studies were conducted in the USA limiting the generalization of the results to the global population. A meta-analysis could not be performed due to study heterogeneity, and publication bias was not assessed due to the small number of available studies for each comparison. Furthermore, this study findings are in-line with previously conducted study on the efficacy and time sensitivity of human amnion/chorion membrane treatment in patients with diabetic foot ulcers which concluded that when amniotic membranes were combined with standard care diabetic foot ulcers healed significantly faster than standard care alone [18]. However, we recommend that further prospective randomized control trials with larger population with long term follow-up have to be performed for better evidence. The current evidence suggests the use of amniotic membrane preparations for resistant diabetic foot ulcers can achieve relatively fast wound closure rates.

\section{Conclusions}

According to our review the current studies summarize reliable evidence to suggest reduction in healing time with amniotic membrane preparations in the treatment of refractory chronic diabetes foot ulcers compared to conventional methods.

\section{Abbreviations}

DFU: Diabetic foot ulcer; dHACM: Dehydrated human amniotic chorionic membrane; DM: Diabetes mellitus; HAA: Human amniotic allograft membrane; NA: Not available; PDGF: Platelet Derived Growth Factors; PRP: Platelet-rich Plasma; RCT: Randomized control trials; SD: Standard deviation; SOC: Standard of care; USA: Untied States of America.

\section{Acknowledgements \\ None.}

Authors' contributions

$\mathrm{KL}$ wrote the first draft of the manuscript with contributions from $\mathrm{OB}$ and $\mathrm{DH}$. All authors read and approved the final manuscript.

Funding

No funding for this project.

Availability of data and materials

Not applicable.

Ethics approval and consent to participate

Not applicable.

Consent for publication

Not applicable.

Competing interests

Authors declare that there are no competing interests.

Received: 20 May 2020 Accepted: 1 February 2021

Published online: 15 February 2021

\section{References}

1. Thompson P, et al. Comparing human amniotic allograft and standard wound care when using total contact casting in the treatment of patients with diabetic foot ulcers. Adv Skin Wound Care. 2019;32(6):272-7. 
2. Zelen CM, et al. A prospective randomised comparative parallel study of amniotic membrane wound graft in the management of diabetic foot ulcers. Int Wound J. 2013;10(5):502-7.

3. Zubair M. Prevalence and interrelationships of foot ulcer, risk-factors and antibiotic resistance in foot ulcers in diabetic populations: a systematic review and meta-analysis. World J Diabetes. 2020;11(3):78.

4. Harris-Hayes $M$, et al. The role of physical therapists in fighting the type 2 diabetes epidemic. J Orthopaed Sports Phys Therapy. 2020;50(1):5-16.

5. Tettelbach $W$, et al. A confirmatory study on the efficacy of dehydrated human amnion/chorion membrane dHACM allograft in the management of diabetic foot ulcers: a prospective, multicentre, randomised, controlled study of 110 patients from 14 wound clinics. Int Wound J. 2019;16(1):19-29.

6. Driver VR, de Leon JM. Health economic implications for wound care and limb preservation. J Manag Care Med. 2008;11(1):13-9.

7. Ya-Na S, et al. Human amniotic membrane allograft, a novel treatment for chronic diabetic foot ulcers: a systematic review and meta-analysis of randomised controlled trials. Int Wound J. 2020;17(3):753-64.

8. Phil $\mathrm{H}$, et al. Age-related macular degeneration and low-vision rehabilitation: a systematic review. Can J Ophthalmol. 2008:43(2):180-7.

9. DiDomenic LA, Orgill DP, Galiano RD, et al. Aseptically processed placental membrane improves healing of diabetic foot ulcerations: prospective, randomized clinical trial. Plast Reconstr Surg Glob Open. 2016:4(10):e1095.

10. Snyder RJ, Shimozaki K, Tallis A, et al. A prospective, randomized, multicenter, controlled evaluation of the use of dehydrated amniotic membrane allograft compared to standard of care for the closure of chronic diabetic foot ulcer. Wounds. 2016;28(3):70-7.

11. Zelen CM, Gould L, Serena TE, et al. A prospective, randomised, controlled, multi-centre comparative effectiveness study of healing using dehydrated human amnion/corion membrane allograft, bioengineered skin substitute or standard of care for treatment of chronic lower extremity diabetic ulcers. Int Wound J. 2015;12(6):724-32.
12. Zelen CM, Serena TE, Snyder RJ. A prospective, randomised comparative study of weekly versus biweekly application of dehydrated human amnion/chorion membrane allograft in the management of diabetic foot ulcers. Int Wound J. 2014;11(2):122-8.

13. Lavery LA, Fulmer J, Shebetka KA, et al. The efficacy and safety of Grafix for the treatment of chronic diabetic foot ulcers: results of a multicentre, controlled, randomised, blinded, clinical trial. Int Wound J. 2014;11(5):554-60.

14. Valiente MR, Nicolás FJ, García-Hernández AM, Fuente Mora C, Blanquer M, Alcaraz PJ, Almansa S, Merino GR, Lucas MDL, Algueró MC, Insausti CL, Piñero A, Moraleda JM, Castellanos G. Cryopreserved amniotic membrane in the treatment of diabetic foot ulcers: a case series. J Wound Care. 2018;27(12):806-15.

15. Abdo RJ. Treatment of diabetic foot ulcers with dehydrated amniotic membrane allograft: a prospective case series. J Wound Care. 2016;25(Sup7):S4-9.

16. Raphael A. A single-centre, retrospective study of cryopreserved umbilical cord/amniotic membrane tissue for the treatment of diabetic foot ulcers. J Wound Care. 2016;25(Sup7):S10-7.

17. Kirsner RS, Sabolinski ML, Parsons NB, Skornicki M, Marston WA. Comparative effectiveness of a bioengineered living cellular construct vs a dehydrated human amniotic membrane allograft for the treatment of diabetic foot ulcers in a real world setting. Wound Repair Regen. 2015;23(5):737-44.

18. Irakoze $L$, et al. Efficacy and time sensitivity of amniotic membrane treatment in patients with diabetic foot ulcers: a systematic review and meta-analysis. Diabetes Therapy. 2017;8(5):967-79.

\section{Publisher's Note}

Springer Nature remains neutral with regard to jurisdictional claims in published maps and institutional affiliations.
Ready to submit your research? Choose BMC and benefit from:

- fast, convenient online submission

- thorough peer review by experienced researchers in your field

- rapid publication on acceptance

- support for research data, including large and complex data types

- gold Open Access which fosters wider collaboration and increased citations

- maximum visibility for your research: over $100 \mathrm{M}$ website views per year

At BMC, research is always in progress.

Learn more biomedcentral.com/submissions 